\title{
Gradhiva
}

GRADHIV

Revue d'anthropologie et d'histoire des arts

$3 \mid 2006$

Du Far West au Louvre : le musée indien de George

Catlin

\section{Présentation : Catlin vu d'Europe}

Daniel Fabre

\section{OpenEdition}

Journals

Édition électronique

URL : http://journals.openedition.org/gradhiva/89

DOI : 10.4000/gradhiva.89

ISSN : 1760-849X

Éditeur

Musée du quai Branly Jacques Chirac

Édition imprimée

Date de publication : 15 juin 2006

Pagination : 5-15

ISBN : 2-915133-26-3

ISSN : 0764-8928

Référence électronique

Daniel Fabre, «Présentation: Catlin vu d'Europe », Gradhiva [En ligne], 3 | 2006, mis en ligne le 10 juin

2009, consulté le 21 septembre 2020. URL : http://journals.openedition.org/gradhiva/89; DOI :

https://doi.org/10.4000/gradhiva.89

Ce document a été généré automatiquement le 21 septembre 2020.

(c) musée du quai Branly 


\title{
Présentation : Catlin vu d'Europe
}

\author{
Daniel Fabre
}

\section{NOTE DE L'ÉDITEUR}

Ce dossier reflète, en partie, les activités de l'Atelier Catlin, tenu à partir de juin 2004 au sein du Laboratoire d'anthropologie et d'histoire de l'institution de la culture (LAHIC CNRS / EHESS / ministère de la Culture et de la Communication).

Je remercie Claude Macherel pour sa lecture attentive de ce texte.

"Comme je descendais les Fleuves impassibles, Je ne me sentis plus guidé par les haleurs : Des Peaux-Rouges criards les avaient pris pour cible

Les ayant cloués nus aux poteaux de couleur.» Arthur Rimbaud, Le Bateau ivre, 1871. 
George Catlin, Máh-to-tóh-pa, Quatre-Ours en tenue d'apparat, 1845. Portrait réalisé sur commande du roi Louis-Philippe suite à la représentation donnée par les lowas au Louvre en avril 1845.

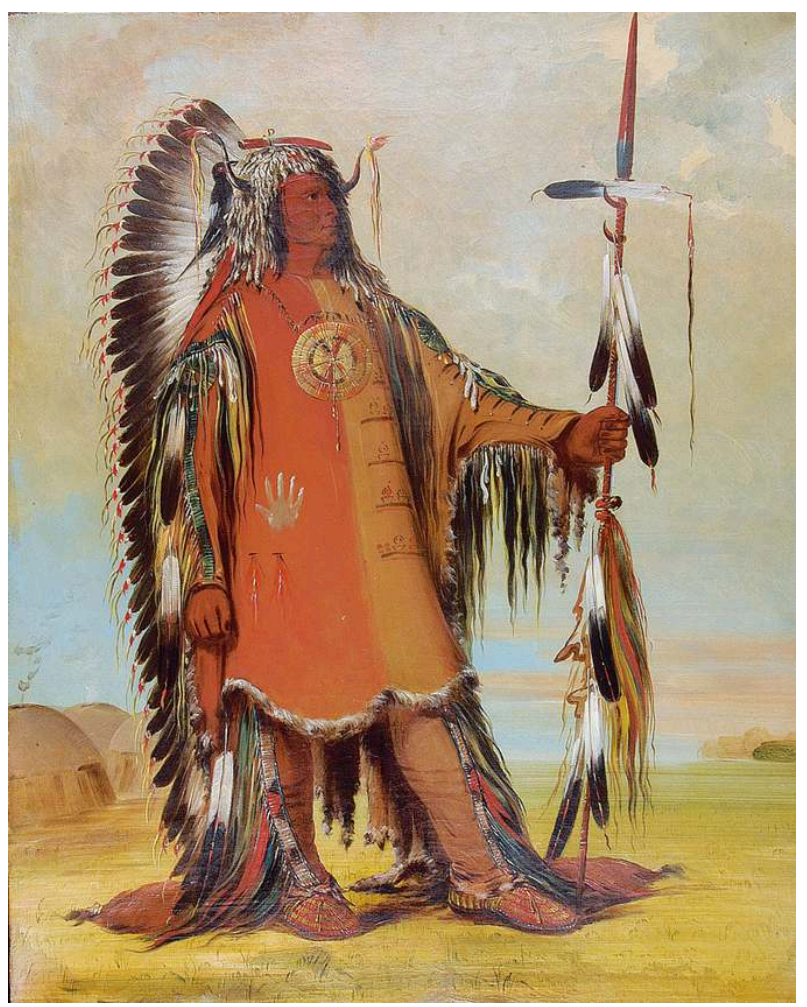

(c) musée du quai Branly

1 L'ouverture, le 6 septembre 2002, à Washington D.C. de l'exposition George Catlin and His Indian Gallery, formée pour l'essentiel des tableaux conservés par la Smithsonian Institution, fut saluée comme un événement d'autant plus notable que depuis plus d'un siècle, la plus grande partie de cette collection n'avait pas eu l'honneur d'une présentation digne d'elle au sein de l'institution qui l'avait sauvée de la destruction en 1879. Redécouvert, ressuscité, tel apparaissait le Musée indien, selon un scénario en tous points conforme à celui de sa première vie. Le musée complet, constitué au terme de six saisons passées dans les plaines du Middle West à visiter une quarantaine de tribus, riche de ses cinq cents tableaux et de ses centaines d'objets, fut présenté pour la première fois en septembre 1837 à New York. Il suscita l'engouement du public mais non celui du gouvernement américain qui, dans l'esprit de Catlin, aurait dû en faire un établissement national. Il embarqua donc son musée pour l'Europe en novembre 1839, l'ouvrit et l'enrichit en Grande-Bretagne (1840-1844) et à Paris (1845), puis, après une brève réapparition londonienne, se le vit littéralement arraché en 1852.

2 Emprisonné quelques semaines pour dettes à Londres, Catlin avait été contraint de vendre la totalité de sa Gallery à un ingénieur, Joseph Harrison, qui se contenta d'entasser cadres et objets (costumes, wigwams, outils, armes, calumets, etc.) dans un des entrepôts de son usine de locomotives, à Philadelphie. Dès lors, enlevée à son auteur, l'œuvre fut ensevelie et oubliée. Certes, Catlin reprendra par épisodes ses voyages dans les années 1850 (Amazonie, Andes, Mexique, Côte ouest), dessinant sans répit, sur cartoons, ses nouvelles rencontres amérindiennes et même ses images perdues. Mais à sa mort en 1872, deux ans après être rentré d'Europe, il ne soupçonnait pas que son musée allait refaire bientôt surface. Après la disparition de Harrison, son 
très négligent sauveur, sa veuve, contactée, fit faire des recherches et l'on exhuma littéralement, à la pelle, de beaux restes de l'Indian Gallery: les trois quarts des tableaux mais seulement quelques objets remontèrent au jour. La Smithsonian Institution, bénéficiaire du legs, les accueillit d'autant plus volontiers que son propre «Musée indien », constitué par le peintre John Mix Stanley dont on avait fait le grand rival de Catlin, était parti en fumée lors d'un grand incendie, en $1865^{1}$. Retour ambigu cependant puisque la collection, une fois pieusement inventoriée par Thomas Donaldson $^{2}$ qui voua six années de sa vie à cette tâche, fut rarement et très partiellement exposée, à titre de spécimen documentaire, avant de rejoindre les fonds immenses de l'Institution, suscitant quelques intérêts érudits, entrecoupés de longues éclipses.

3 En 2002, le monde ouvrait donc les yeux sur Catlin pour la troisième fois. Mais quelque chose avait changé depuis la résurrection posthume: ces peintures des visages, des costumes, des corps, des lieux d'existence, des activités quotidiennes et des rituels des Indiens des Plaines dans les années 1830 n'étaient plus simplement alignées en forme de frise sous les plafonds d'une salle de conférences ou d'exposition, ils étaient rendus à la complétude - depuis longtemps démembrée - du projet de leur créateur dont le visiteur pouvait mesurer à quel point il l'avait hanté toute sa vie. Du coup, l'ensemble consacrait son auteur comme l'un des peintres de la genèse américaine puisque les débuts de la peinture "nationale » avaient coïncidé avec l'expansion de la société blanche, irrésistible et destructrice, depuis ses foyers côtiers, à l'Est du continent. Geo. Catlin $^{3}$, l'artiste, aurait donc signé une vision enchantée mais exacte des sociétés indiennes juste avant leur crépuscule qu'il pressentait avec mélancolie et indignation. Ce point de vue restitué rencontra l'assentiment du public qui se pressa à l'exposition lors de sa tournée de trois ans à travers le pays. Il cristallisait une évolution amorcée depuis trois ou quatre décennies qui faisait de Catlin, pourvoyeur devenu anonyme de tant d'images répandues de l'Ouest sauvage, non seulement un ethnographe avant la lettre mais le visionnaire d'une histoire devenue commune.

Pourtant, les débats d'interprétation suscités par cet événement, les réflexions que ses promoteurs avancent ou suggèrent, la situation précisément évoquée dans laquelle fut conçue, il y a cent soixante-dix ans, l'Indian Gallery, et surtout le fait, trop vite souligné, que la réception de l'œuvre complet de Catlin fut, en son temps, essentiellement européenne, sont autant d'incitations à revenir sur cette création et sur son impact. Sans prétendre rendre compte exhaustivement du très long exil de Catlin - trente années, avec puis sans son musée, mais toujours ses Indiens dans l'esprit et sous le pinceau -, Gradhiva a souhaité repenser cet événement. Pour une raison au moins : sous la forme très particulière où il conjoignit, au temps de sa splendeur parisienne, 585 peintures, un millier d'objets et des Indiens qui donnaient sur scène une part de leurs rites - musiques, danses, chants et discours -, le Musée indien a constitué la première grande exposition anthropologique visible en Europe. Son inventeur rencontra les personnalités et les cercles londoniens et parisiens de l'ethnologie naissante, pendant que son art nourrissait d'une altérité toute neuve le premier " primitivisme », celui des " artistes » de la seconde génération romantique. Qu'il ne reste rien de tout cela, ou si peu, invite à s'interroger sur un principe d'effacement, de dénégation ou de méconnaissance : en France, dans les musées où il est exposé et partagé, le savoir ethnologique gomme nécessairement, semble-t-il, ses expressions antérieures, pensant ainsi rafraîchir son regard. 


\section{Dissonances}

5 Le 20 décembre 2002, Michael O'Sullivan, chargé de la critique culturelle au Washington Post, lançait une note discordante dans le concert d'éloges qui avaient accueilli l'inauguration de George Catlin and His Indian Gallery. En situant cette redécouverte dans l'ambiance du « colonial blue », il rappelait, non sans malice, que les mêmes institutions avaient, dix ans plus tôt, présenté au National Museum of American Art (NMAA), une exposition autrement controversée, The West as America, dont Catlin était aussi l'un des protagonistes. Attachés, en cette année 1991, à dénoncer les illusions de l'idéologie yankee et la fabrique nationale des mythes, les commissaires avançaient une proposition générale : à partir des années 1820 , tous les auteurs de portraits d'Indiens et de paysages intouchés auraient purement et simplement "inventé » une sorte d'Arcadie où les peuples amérindiens n'étaient que les figurants d'un tableau idyllique. Incapables de représenter la violence de la conquête, ils auraient mis en scène un état de nature qui devait, fatalement, laisser place à la civilisation triomphante. Sous leur plume, l'éradication des sociétés indiennes n'était pas la raison de la représentation mais bien son effet : l'image funéraire préparait la mise à mort. Les peintres de paysage eux-mêmes n'opéraient-ils pas une prise de possession par le regard, ouvrant la voie aux cartographes d'état-major et préludant à une mise en coupe réglée des territoires ? Parmi ces illusionnistes, complices plus ou moins consentants de la violence de l'Histoire, Catlin occupait une place de choix puisqu'il fut le premier Américain à aller peindre les tribus indiennes "sur le motif». Circonstance aggravante, le commissaire de cette exposition militante n'était autre que William H. Truettner, historien d'art, conservateur au département des Peintures et Sculptures du NMAA et grand spécialiste du peintre de l'Ouest dont il avait donné une très sûre monographie ${ }^{4}$. 
George Catlin, Le Roi Louis-Philippe au Palais des Tuileries recevant M. Catlin et les Indiens lowas, 1845.

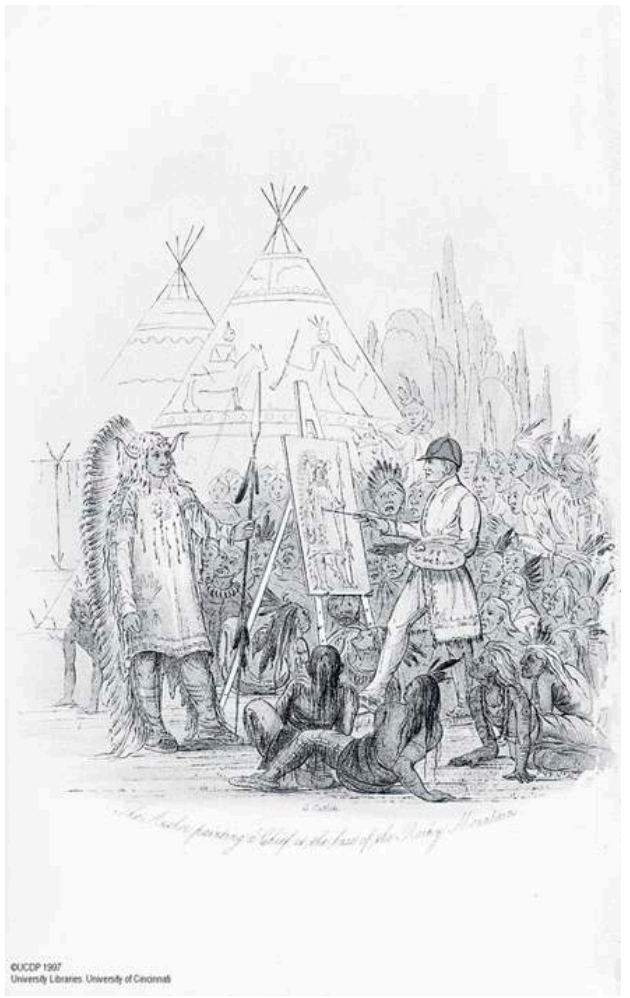

Archives and Rare Books Library, University Libraries, University of Cincinnati

Cette critique s'applique parfaitement à un peintre comme Frédéric Remington, chroniqueur épique de la conquête ${ }^{5}$. Elle manque son but s'agissant de Catlin, puisqu'elle ne tient aucun compte ni de ses intentions ni des conditions de la représentation picturale dans lesquelles il s'inscrit. Claude Macherel le caractérise exactement, dans ce numéro, comme un « hybride culturel » en vertu d'une généalogie et d'une enfance, généralement à peine évoquées, qui nouent en lui de façon définitive l'expérience des deux mondes ${ }^{6}$. Ensuite, Patricia Falguières rappelle que dès les débuts de sa vocation, quand il décide de partir vers l'Ouest, il se veut " peintre d'histoire », un genre dont le portrait est une des expressions ; adepte donc d'une nouvelle alliance de la forme et de la fonction picturales que les Anglais formulent et expérimentent depuis un demi-siècle.

7 La relecture politique de l'œuvre avait été précédée d'une autre réduction, qui retenait, non les tableaux du peintre et les scènes du muséographe, mais les performances du showman. On prétendit nous révéler, sous la noblesse pédagogique du musée, un divertissement qui préfigurait le West Wild Show auquel William Cody, alias Buffalo Bill, donnera, un demi-siècle plus tard, une renommée populaire mondiale ${ }^{7}$. Là encore, autour de 1840, l'expansion de la popular culture américaine, c'est-à-dire de la première culture de masse au sens contemporain, joua à Catlin un mauvais tour qui découle d'un voisinage entre son Indian Gallery et de nouvelles et florissantes entreprises, celle de P. T. Barnum en particulier. La polysémie du mot "musée " (museum, gallery) a nourri l'ambiguïté. Plusieurs décennies durant, il a désigné le rassemblement des collections, de haute valeur esthétique et nationale, dans un lieu prestigieux voisin du pouvoir formule que le Louvre incarne idéalement - aussi bien que quantité de spectacles où la 
présentation des œuvres s'accompagne du merveilleux technique le plus actuel ainsi que des effets spéciaux du théâtre, du cirque et de la foire ${ }^{8}$. D'un lieu à l'autre, l'attitude du spectateur fait toute la différence. Dans le musée animé, il peut, il doit exprimer avec exubérance les émotions que le musée statique entrave, en exigeant silence et concentration, des attitudes dévotes qui, à la même époque, ont du mal à s'imposer dans les salles de théâtre et de concert comme elles tarderont longtemps à le faire dans les salles de cinéma. Or, les Londoniens et les Parisiens des années 1840 n'étaient en rien choqués par cette dualité, même si la critique commençait à tracer une frontière étanche entre les deux manières. Baudelaire, amateur des musées au sens moderne dès l'adolescence, n'en reconnaît pas moins la haute valeur du Musée indien de Catlin'. Que quelques puristes londoniens aient traité ce dernier de «bateleur yankee», au nom d'une hiérarchie toute fraîche, ne suffit pas à l'enrôler dans l'alliance du freakness et du business que Barnum, renonçant délibérément à ses premières ambitions esthétiques et pédagogiques, était en train de lancer avec un franc succès ${ }^{10}$.

Une autre critique, plus radicale, exprime mieux encore la singularité catlinienne dès son séjour à Londres. Venant après certaines exhibitions qui connurent une fin lamentable - par exemple celle des Osages à Paris en $1827^{11}$ - Catlin, présentant des Indiens de chair et d'os, incarnerait la première phase des «zoos humains » - qui allaient participer, jusque dans les années 1930, au spectacle des retours d'expéditions et des expositions coloniales $^{12}$. Or, ces parades suscitaient en lui de profondes réticences.

9 L'idée de mettre en scène les Indiens en personne, comme un élément des scènes muséales, n'est pas neuve. Tout porte à croire que le peintre fréquentait à New York, où il se trouvait, le New York Museum que Charles Wilson Peale avait monté sur le modèle de son grand musée de Philadelphie auquel le jeune Catlin doit l'essentiel de sa formation. Or, en 1826, comme une affiche miraculeusement préservée l'atteste, une troupe d'Indiens chippewas (autre nom des Ojibwas) - chiefs, warriors and squaws - se produisait dans ce haut lieu de Broadway. La recette était donc parfaitement connue et Catlin refusa de l'appliquer en s'embarquant pour l'Europe, en novembre 1839, avec seulement son neveu (Theodore Burr Catlin), un homme de confiance (Daniel) et deux ours grizzlis fort mal apprivoisés. Il faut attendre février 1844, alors que son exposition londonienne, à l'Egyptian Hall, n'attire plus autant les foules, pour qu'il accepte de faire monter sur scène une troupe d'Ojibwas venue du Canada sous la conduite d'un jeune impresario passablement aventurier, Arthur Rankin, qui les a déjà fait tourner dans les foires de Toronto et des environs. Après leur départ, au début de l'été 1844 , ce sont des Iowas qui semblent lui tomber du ciel puisqu'il avait, quinze ans auparavant, connu et peint leurs parents près de Saint-Joseph, Missouri. De fait, ils ont été enrôlés et présentés à New York par Barnum, lequel, arrivé à Londres, leur préfère l'exhibition beaucoup plus attractive de Tom Thumb, un nain qu'il déguise en général américain. Dans ses récits, Catlin cachera toujours cet héritage involontaire, façon maladroite de se distinguer d'un concurrent qu'il juge exagérément avide et radicalement étranger à la passion qui le meut ${ }^{13}$. Conduits à Paris, qu'ils enchantent, les Iowas seront bientôt remplacés par d'autres Ojibwas cornaqués par un de leurs pasteurs plus ou moins défroqué, George Henry alias Maun-gwa-daus, dont on lira, traduit par Claudie Voisenat, le "journal de voyage" dans ce numéro. Ils accompagneront Catlin à Bruxelles qu'ils quitteront à l'automne, décimés par la variole. Au total, dix-huit mois, où, de façon très intermittente, Catlin a remplacé les acteurs qui, dans les premiers temps, animaient son musée de leurs pantomimes et autres «tableaux vivants », par de 
«vrais » Indiens qui s'étaient, de leur propre chef parfois, lancés dans le spectacle de leur culture ${ }^{14}$. Façon de raviver l'intérêt pour son musée dont il expliquera en détail qu'elle se révéla finalement déprimante et ruineuse.

10 Ni showman au sens que Barnum donne à ce terme, ni "zoologue » des humanités différentes, Catlin est animé par une ambition unique, constamment remise à flot contre vents et marées : élever en Amérique ou, à la rigueur, en Europe, un mémorial riche de visages singuliers, de scènes prises sur le vif, de paysages ouverts, un monument vibrant de couleurs et de matières qui donne à voir les derniers instants de la vie libre des Indiens ${ }^{15}$. Il ne peut donc justifier la mise en spectacle de l'Autre qu'il réalise sciemment qu'en inversant le sens de la curiosité et de la représentation. Comme le montre Gaetano Ciarcia dans son article, sans avoir lu les Dialogues du baron de La Hontan avec le voyageur huron ou les Lettres persanes de Montesquieu, Catlin fait de ses Indiens, des Iowas en particulier, les visiteurs curieux et attentifs du monde occidental ${ }^{16}$. Il les montre, embarquant pour l'Amérique leur propre trésor, un Musée européen accumulé au fil des ans : malles remplies de livres, d'images et d'objets, cahiers truffés de chiffres et de mots. Trompe-l'œil rhétorique, dira-t-on, mais qui marque nettement la distance entre l'entreprise catlinienne et l'esprit colonial du temps, distance qui justifia explicitement le refus maintes fois opposé par le Congrès américain d'acquérir cette œuvre que la majorité considérait comme une critique radicale de la politique indienne du gouvernement et, plus profondément, des fondements de la civilisation blanche.

11 La récente exposition américaine répliquait donc aux lectures « démystifiantes » de la décennie précédente. Attentive à l'histoire mouvementée de ce qui fut un "musée » avant de devenir une " collection $»^{17}$, elle rendait Catlin à son projet vital, mais il n'est pas sûr que, dans cette juste ambition, elle ait pris la mesure de l'expérience européenne qui vit alterner en un soudain contraste l'éclat et l'oubli.

\section{Les dix glorieuses}

12 Le voyage d'Europe généra, au cours de ses années brillantes (1840-1850), un récit qu'ordonne à chaque pas la figure récurrente d'une inversion positive. Alors que ses voyages dans l'Ouest n'ont jamais abouti, aux États-Unis, qu'à des lettres publiées dans la presse locale et à un catalogue qui ne dépassera pas, en 1838, la quarantaine de pages, l'installation londonienne puis parisienne incite Catlin à composer deux gros ouvrages idéalement symétriques. Le premier, Catlin's Letters and Notes..., édité dès 1841 à compte d'auteur "at the Egyptian Hall», rassemble ses voyages dans le Far West américain, le second dont le titre définitif sera Adventures of the Ojibbeway and Ioway Indians in England, France and Belgium, paraît, encore "chez l'auteur", en 1848; il contient la relation du double séjour de Catlin et des troupes indiennes dans le Far East européen. Chaque livre est illustré de planches lithographiées. En tête de chaque tirage des

Letters, une douzaine d'exemplaires sont rehaussés de couleurs par Catlin, ou par les jeunes peintres (John Cullum, Rosa Bonheur) qu'il recrute à Londres où il a monté un petit atelier très actif. Devant le succès de ses récits et de ses images, Catlin envisage même de redessiner et lithographier l'ensemble de sa Gallery qui sortirait ainsi, par chapitres thématiques, en portfolio, projet dont il ne réalisera, faute d'argent, que le premier volume. Écrire, graver, lithographier, faire imprimer, colorier - des livres, des 
catalogues, des tracts, des affiches -, tout en peignant les portraits de tous les Indiens qui le rejoignent et en dessinant les grands épisodes de leur "tour ", telles sont, avec les lectures qu'il présente dans son musée, les entreprises de Catlin au cours de ces premières années européennes toutes fiévreuses d'action. Ainsi, l'œuvre en cours incarne-t-elle très vite l'autre sens du terme "musée ", que le XIx siècle vient de retrouver, soit «la mise en écriture des œuvres» et, plus largement, «le rassemblement méthodique des savoirs liés à la collection ». Cette ampleur et cette diffusion immédiate, que l'imprimerie rend possible, permettent au peintre de nourrir son plan de reconquête de l'Amérique où il fait, au fur et à mesure, retirer ses livres et ses recueils d'images. Accompagnant cette croissance, l'œuvre de Catlin reçoit en Europe un accueil chaleureux, qui va de l'attention à l'enthousiasme de la part des puissants, des savants et des artistes.

13 Alors que l'accès aux autorités suprêmes exige, dans la démocratique Amérique, des médiations complexes et incertaines et un lobbying ininterrompu, voilà d'un coup Catlin, grâce à son Musée et aux troupes indiennes qui s'y agrègent, reçu par les souverains avec aménité, déférence, amitié parfois. Victoria à Londres, Louis-Philippe à Paris, Léopold $\mathrm{I}^{\text {er }}$ à Bruxelles, Ferdinand-Guillaume IV à Berlin accueillent, conformément à une très ancienne prérogative des monarchies européennes, non tant le promoteur de curiosités exotiques que l'ambassadeur du Nouveau Monde qui introduit les chefs de nations indiennes. À Bruxelles, dans sa période la plus noire, après 1852, Catlin ne cessait de rappeler ces instants de familiarité où le voyageur, gris de la poussière des routes, était reçu à l'instant dans les palais royaux. Cette nostalgie du solitaire efface la désillusion de l'entrepreneur qui avait confondu politesse des rois et promesse de mécénat, mais elle donne la mesure du plaisir orgueilleux d'être reconnu qui avait embelli son installation européenne.

Parallèlement, le musée catlinien a un retentissement dans le monde du savoir qui est en train d'organiser le territoire des disciplines. Lors de ses premières apparitions dans les villes américaines, Catlin avait, certes, reçu des soutiens intellectuels ; quelques-uns appréciaient la véracité documentaire de ses travaux. Pourtant la campagne orchestrée contre lui par Henry Rowe Schoolcraft, l'ethnographe officiel, voulait que ses productions soient embellies, fantaisistes, voire complètement inventées, ce qui contribua à l'échec répété de ses pétitions auprès du Congrès ${ }^{18}$. On comprend mieux alors pourquoi, au cours de ses années européennes, Catlin prend soin de nouer des liens avec les savants et leurs institutions. À Londres, dès 1843, il accepte, pour la seule et unique fois de sa vie, d'illustrer une œuvre qui n'est pas la sienne. Il s'agit du testament scientifique du grand James C. Prichard, The Natural Study of Man, aussitôt traduit en français et publié à Paris, pour lequel il donne dix portraits en couleur et six dessins au trait ${ }^{19}$. Parmi les visites d'importance dans ce registre, il retiendra surtout celle d'Alexander von Humboldt qu'il semble avoir reçu à Londres et à Paris et qu'il sollicitera dans sa retraite prussienne, à maintes reprises, au cours de ses années obscures. Il faut ajouter à ces noms de premier plan celui de Daniel Wilson, Écossais devenu canadien, auteur d'une importante théorie "atlantidienne " de la continuité culturelle transatlantique ${ }^{20}$; et surtout l'abbé Brasseur de Bourbourg, spécialiste majeur des cultures et des écritures précolombiennes que Catlin approche dans les années 1850 et qui ne lui ménagera jamais son soutien. Présenter les Iowas à l'Académie des sciences à Paris, devenir membre de la Société d'ethnologie fondée dans cette ville en $1838^{21}$, développer devant l'Ethnological Society de Londres le plan d'un Musée de 
l'Humanité itinérant, dont Patricia Falguières nous propose ici une traduction, répondre à la demande d'expertise d'une pictographie iroquoise que lui adresse la Bibliothèque royale, sont autant d'épisodes qui consolent Catlin des réticences et des humiliations américaines ${ }^{22}$. Dans les années 1860, il tentera, avec un succès moyen, de ranimer ce réseau d'accointances savantes pour défendre ce qu'il considère comme ses apports majeurs à l'ethnographie : la description de la cérémonie d'initiation O-kee-pa qu'il a vue en 1832 chez les Mandans, décimés peu après, et celle des carrières de Pipe stone où les tribus viennent de très loin extraire la pierre rouge dans laquelle on sculpte les calumets; deux contributions que la critique de ses adversaires avaient, très injustement, mises en pièces ${ }^{23}$. Même s'il s'est risqué à des considérations générales sur la parenté des cultures, Catlin n'a jamais eu la prétention de participer directement à la construction d'une discipline anthropologique encore balbutiante. Mais l'un de ses authentiques fondateurs, Henry Lewis Morgan, sera très proche de lui par ses expériences formatrices et par son attention respectueuse à l'égard du monde indien. Par une singulière ironie du sort, on doit à Morgan la description d'une visite, en 1859, aux Iowas sédentarisés où son interlocuteur principal n'est autre que Nuage Blanc, le chef des Iowas présents à Londres et à Paris, ou l'un de ses proches parents ${ }^{24}$.

«Les Ojibbeways de Catlin visitant Londres », Punch, 20 avril 1844.

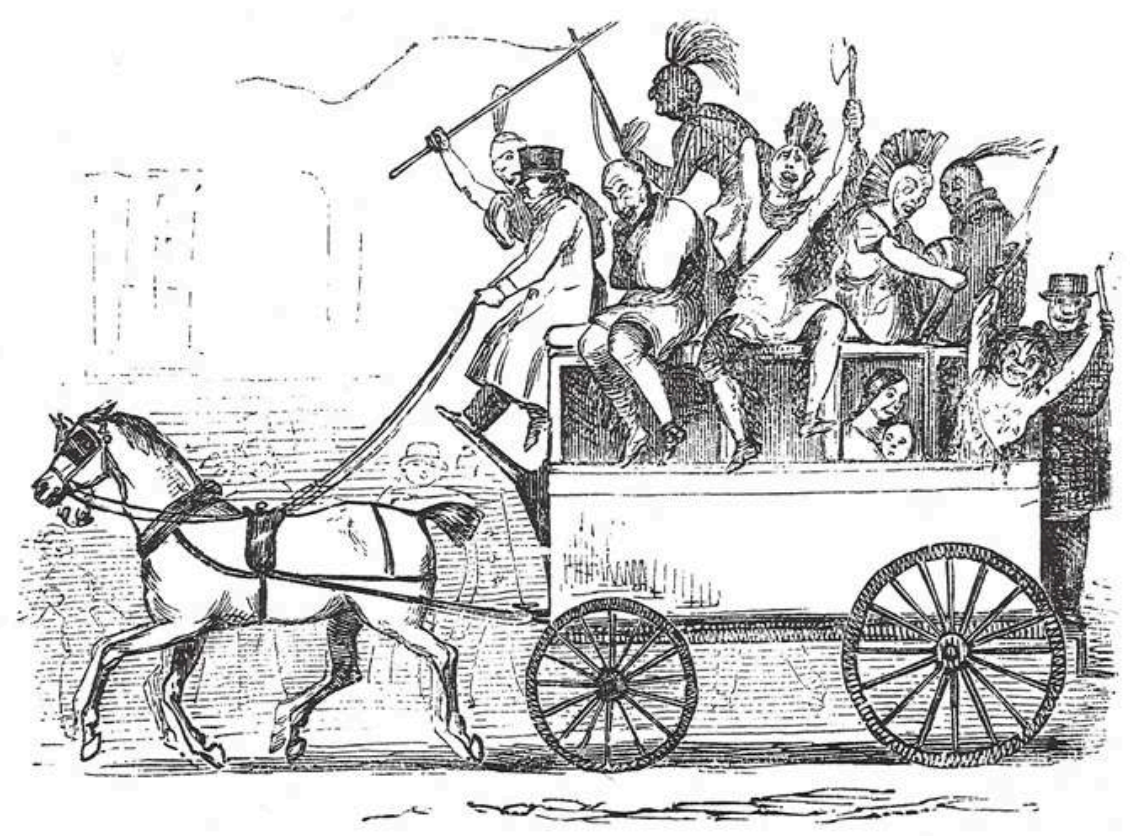

droits réservés

Le regard sur Catlin artiste, à peine esquissé dans la période américaine, prendra, quant à lui, forme en Europe dans l'opposition entre Londres et Paris. Peu reconnu comme tel outre-Manche, il sera célébré comme un peintre de la modernité dans la capitale de l'art occidental, sans que lui-même soit pleinement conscient de l'impact de son œuvre sur la jeune génération qui formule à Paris l'esthétique d'avant-garde ${ }^{25}$. Il a lu les articles enthousiastes de George Sand et de Théophile Thoré, il ne dit rien de Nerval, de Gautier, de Champfleury ni de Baudelaire. Arlette Sérullaz nous rappelle, en présentant 
ici un précieux carnet à peine redécouvert, que Delacroix est venu croquer ses Indiens, et nous savons que d'autres peintres passèrent de longues journées auprès d'eux, incitant leur confrère à envoyer des œuvres au Salon de 1846. Il fallut attendre longtemps pour que ce Catlin créateur retraversât l'Atlantique. L'invention du "luminisme », dans les années 1970, par quelques critiques américains, comme école picturale nationale, née du face-à-face de l'art et d'une «nature » jamais représentée auparavant, doit sans doute quelque chose à l'acclamation des «ciels» de Catlin par Baudelaire et ses amis ${ }^{26}$.

16 L'accueil fait aux images de Catlin se mesure également ailleurs. Frédéric Maguet montre ici que les adaptations populaires de l'imagerie catlinienne, qui vont des bois coloriés d'Épinal à la lithographie pour magazine, en passant par les xylogravures des catalogues, ont abouti, en France, à la métamorphose de la figure des Indiens américains. Après les fantaisistes Incas emplumés, hérités du xvIII ${ }^{\mathrm{e}}$ siècle, après l'interprétation néoclassique des héros de Chateaubriand et des vrais Osages de 1827, Catlin renouvelle les visages et les scènes, introduisant à la fois la violence de l'action et l'infini paisible de la Prairie. À "l'enfant amoureux de cartes et d'estampes » (Baudelaire, Le Voyage), il offrait les «fleuves impassibles", les «Peaux-Rouges criards » et les «poteaux de couleur » que Rimbaud trouva dans sa lecture avide des revues illustrées où Catlin réapparaissait - est-ce fortuit? - quelques mois avant les dérades du Bateau ivre ${ }^{27}$.

\section{Le principe d'amnésie}

17 Le nom de Catlin se perdit dans la crue des images européennes, au moment où l'édition originale - et la traduction française監- de sa superbe autobiographie d'enfant et de voyageur devenait un succès des bibliothèques de gare, avant d'être un titre maintes fois réédité de la "Bibliothèque rose" (Catlin 1863). Ce passage à une sorte d'immortalité visuelle et narrative, payé au prix de l'anonymat, aurait pu être une retombée de la gloire. Ce fut l'inverse qui arriva : l'œuvre de Catlin, la totalité insécable que constitue son musée à facettes multiples, connut en Europe un effacement très rapide qui ne tient pas seulement aux convulsions de l'époque et à la singularité de son destin. Contribuent à son élimination en tant qu'auteur, à la fois des changements radicaux de la façon de faire œuvre muséale et une sorte de principe inaperçu qui impose l'immolation active de toute mémoire quand il s'agit de raviver, par une nouvelle rencontre de l'Autre, les manières occidentales de connaître et d'absorber la diversité des cultures et des arts.

En acceptant avec empressement, au cours de leurs rencontres de l'année 1845, de peindre, à la demande de Louis-Philippe, quatorze tableaux indiens puis vingt-sept autres destinés au Musée historique de Versailles, représentant l'exploration du delta du Mississippi par Cavelier de La Salle, Catlin semblait faire un pas vers l'intégration dans le marché des commandes d'État, qui, au même moment, faisait vivre Delacroix. Pourtant, accroché à l'idée qu'un jour prochain son œuvre trouverait un port définitif, il ne vendit jamais ses tableaux existants, comme l'aurait fait n'importe quel peintre. Il préféra se copier lui-même, quitte à renouveler ses compositions en prélevant ici et là dans la matière visuelle amassée jadis dans l'Ouest. Il n'entra donc pas dans le marché de l'art puisque ses productions étaient volontairement dépouillées du caractère unique qui en ferait la valeur et en fonderait l'aura. Il réservera toujours cette unicité 
au musée entier, qu'il accrut en toute occasion, en peignant par exemple chaque Indien qui le rejoignait en Europe.

Ce rêve d'immortalisation d'un Tout aurait-il pu prendre corps ailleurs? La fin européenne du Musée indien, redevenu pour quelque temps Indian Gallery à Londres, coïncide avec l'éclosion des Expositions universelles, la première prenant place en 1851, dans le fameux Crystal Palace. Catlin a caressé l'idée d'en faire, pour quelques mois, le lieu où son œuvre serait révélée au monde, mais il eût fallu, pour cela, que l'État américain l'élise comme l'un de ses artistes, et ce ne fut pas le cas. Aussi présenta-t-il seulement deux mannequins costumés en Indiens, tout en déambulant au milieu de la foule avec un groupe d'Iroquois rencontrés là, ceci dans l'espoir d'attirer l'attention sur son projet de Museum of Mankind dans lequel il croyait tenir une nouvelle planche de salut. Or, dans une scène dont le caractère prémonitoire est saisissant, une femme ivre brise les figures indiennes et les Iroquois dénoncent sur le champ l'esprit du mal dans l'eau-de-feu des Blancs ${ }^{28}$. En 1855, l'Exposition est à Paris et Catlin n'y est plus. Son musée vendu, il court les Amériques, cependant qu'un de ses admirateurs et imitateurs, Paul Kane, est présent dans le pavillon du Canada, avec quelques tableaux et des objets ethnographiques. De fait, le peintre des Indiens, à Paris et à Londres, n'est plus Catlin, mais Kane. Tout en suivant la piste de son aîné, rencontré à Londres en 1842 à la fin de son "grand tour " en Europe, Kane a bien compris que le rêve d'un musée unique et complet n'était plus de ce temps. Lui-même, qui ne cite pas Catlin dans son récit de voyage, accepta de mener une carrière normale en vendant au détail ses tableaux à la bourgeoisie et aux musées de son pays ${ }^{29}$.

Le retour de l'Exposition universelle à Paris, en 1878, coïncide avec la décision de doter la France d'un grand musée ethnographique au Trocadéro, palais hispano-mauresque construit tout exprès sur la colline de Chaillot. Comme la France, républicaine et coloniale, a décidé de déposer là tous les exotica des anciennes collections royales, on aurait pu s'attendre à voir ressurgir, au moins, les quatorze tableaux de Catlin commandés par Louis-Philippe. Il n'en est rien, ce qui donne la mesure de l'effacement alors même qu'une nouvelle fièvre indienne gagne le roman d'aventures, l'image de masse et des peintres de renom, dont Rosa Bonheur qui connut Catlin dans sa jeunesse. Le contraste est saisissant entre les multiples rééditions de Ma vie chez les Indiens dans la bibliothèque rose et la maigre notice du Grand Larousse, minutieuse mémoire du siècle, qui retient Catlin comme le géologue qui a donné son nom à la catlinite, pierre rouge des carrières de Pipe stone ${ }^{30} \ldots$

Du Trocadéro au musée de l'Homme, en 1936, rien n'éveille cette mémoire. Le lecteur des œuvres esthétiques de Baudelaire qui aurait cherché dans ce lieu des images à mettre sous le nom vénéré de Catlin, serait rentré bredouille. Pourtant, les traces étaient là, présentes. Il fallut, dans les années 1990, qu'un curieux déroule un paquet de toiles gisant dans les réserves de la bibliothèque du musée et reconnaisse qu'il y avait là, mystérieusement échoués et préservés, une douzaine de tableaux de Catlin (Rotschi 1993). Nous savons aujourd'hui qu'ils furent peints en 1846 pour le « roi des Français » qui aimait l'Amérique indienne. Discrètement restaurés, en voilà certains visibles comme ils ne l'ont jamais été dans les salles du musée du quai Branly. Et ce, au moment où la reconnaissance de Catlin "artiste national américain ", jointe à l'extrême rareté de ses peintures originales sur le marché, en fait un peintre inaccessible, dont la moindre lithographie rehaussée à la gouache s'échange aujourd'hui à prix d'or. 
Ce Catlin-là, transfiguré par sa conversion en icône de la culture nord-américaine, pourrait être le sujet d'un autre travail. Ce numéro de Gradhiva a choisi de porter plutôt l'accent sur la genèse d'une création et d'une réception, afin de conjurer, pour la France et l'Europe, la perte de mémoire. Le faire au moment où s'ouvre à Paris un nouveau musée consacré aux cultures et aux arts dits "premiers", introduit une dimension méditative dans l'euphorie. Comme dans ces banquets antiques évoqués par Montaigne, où une momie exposée au lieu du festin rappelait au souverain sa condition mortelle, il nous a semblé nécessaire de tempérer l'idée d'un «musée éternel » ${ }^{31}$. En France plus qu'ailleurs - mais pourquoi? - aucune entreprise de rassemblement des altérités culturelles n'a pu vivre, réellement vivre, au-delà d'une génération. Selon Van Gennep et Apollinaire, le Trocadéro était poussiéreux en 1910 ; le musée de l'Homme commença à donner des signes d'obsolescence dès les années 1960 ; le musée des Arts et Traditions populaires n'a pas joui de plus de trente ans d'existence. Dans ces lieux où le discours compréhensif prime toujours des œuvres dont la totalisation est forcément partielle, provisoire et inlassablement réarticulée, tout se passe comme si chaque génération, exprimant sa situation historique, devait refondre le miroir pour y projeter ses expériences de la rencontre. Alors même que le souci savant, et donc cumulatif, est partout affiché, la figure exemplaire et la logique à l'œuvre sont celles du créateur moderne qui doit nécessairement refaire par lui-même les pas que d'autres firent, en repassant par le commencement. Le destin du Musée indien de Catlin semble pousser à l'extrême cette contradiction, lui qui voulait conjoindre, sans hiérarchie et en une fusion complète, œuvre de l'art et œuvre de savoir. Refusant de choisir entre ces deux régimes d'existence, il s'est livré au noir de l'oubli et à la périodicité des renaissances. N'est-ce pas, pour cet inventeur toujours à contretemps, la seule façon de continuer à être au monde?

\section{BIBLIOGRAPHIE}

Textes de George CATLIN

1838 Catalogue of Catlin's Indian Gallery. New York, Piercy \& Reed.

1841 Letters and Notes on the Manners, Customs and Conditions of the North American Indians, written during eight years's travels amongst the wildest tribes of Indians in North America in 1832, 33, 34, 35, 36 37, 38 and 39 by Geo. Catlin in two volumes, with four hundred illustrations carefully engraved from the original paintings. Londres, G. Catlin, Egyptian Hall, 2 vol.

1844 Catlin's North American Indians Portfolio, Hunting Scenes ans Amusements of the Rockey Mountains and Prairies of America, from drawings and notes of the author made during eight years of travels amongst forty-eight of the wildest tribes of savages in North America. Londres, G. Catlin, Egyptian Hall, Picadilly, printed by Day \& Hague.

1848 Catlin's Notes of Eight Years Travels and Residence in Europe, with His North American Indian Collection, with anecdotes and incidents of the travels and adventures of three different parties of American 
Indians whom he introduced to the court of England, France and Belgium, by Geo. Catlin. London, G. Catlin, 2 vol.

1852 Adventures of the Ojibbeway and Ioway Indians in England, France and Belgium, notes of eight years travels and residence in Europe with his North American Indian collection, by Geo. Catlin. London, G. Catlin, 2 vol. (réimpression du précédent sous ce nouveau titre).

1861 Life Amongst the Indians : a Book for youth by George Catlin. Londres, Sampson Low, Son \& co.

1863 La Vie chez les Indiens. Scènes et aventures de voyage parmi les tribus des deux Amériques, traduit et annoté par F. de Lanoye et illustré de 25 gravures sur bois. Paris, Louis Hachette et Cie (réédité cinq fois dans la «Bibliothèque rose illustrée » entre 1867 et 1890).

1867 O-kee-pa, a Religious Ceremony, and other customs of the Mandans. Londres, Truebner.

1869a "Excursions parmi les tribus indiennes des bassins de la Columbia et du Haut-Missouri ", d'après M. G. Catlin. 1855, Le Tour du monde, tome $19: 145-160$.

1869b « II O-kie-pa. Cérémonie religieuse et expiatoire des Indiens Mandans », Le Tour du monde, tome $19: 161-176$.

1959 Les Indiens de la Prairie. Dessins et notes sur les mours, les coutumes et la vie des Indiens de l'Amérique du Nord, par George Catlin, 1792-1872, traduit par Fance Frank et Alain Gheerbrant. Paris, Club des Libraires de France.

1979 Indian Art in Pipestone : George Catlin Portfolio in the British Museum, édité par J. C. Ewers. Londres, British Museum Publications.

1992 Les Indiens d'Amérique du Nord, trad. Danièle et Pierre Bondil. Paris, Albin Michel, « Terre indienne ».

1996 Catlin's O-kee-pa: Mandan Culture and Ceremonial. The George Catlin O-kee-pa.Manuscript in the British Museum, éd. par Colin Taylor, Wyk auf Föhr, Verlag für Amerikanistik.

ADAMS, Bluford

1997 e pluribus Barnum : The Great Showman and the Making of U.S. Popular Culture. MinneapolisLondres, University of Minnesota Press.

Anonyme

1855 Le Canada et l'Exposition universelle de 1855. Toronto, John Lovell.

BANCEL, Nicolas et BLANCHARD, Pascal, éd.

2002 Zoos humains. De la Vénus hottentote aux reality shows. Paris, La Découverte.

BARNUM, Phineas Taylor

1873 Struggles and Triumphs or Forty Years'Recollections. Buffalo, Warren \& Johnson.

1983 Selected Letters of P. T. Barnum, A. H. Saxon éd. New York, Columbia University Press.

BOGDAN, Robert

1988 Freak Show. Presenting Human Oddities for Amusement and Profit. Chicago, The University of Chicago Press.

BRESC-BAUTIER, Geneviève

1999 « Les Musées du Louvre au XIX ${ }^{e}$ siècle : les collections archéologiques et ethnologiques dans le conservatoire de l'art classique », Cahiers de l'école nationale du Patrimoine : 53-70. 
BUDDLE, Kathleen

2004 « Media, Markets and Powwows. Matrices of Aboriginal Cultural Mediation in Canada », Cultural Dynamics, vol. 16, $\mathrm{n}^{\circ} 1: 29-69$.

CERISIER, Emmanuel

2004 George Catlin, peintre des Indiens. Paris, L'École des loisirs.

CooK, James W.

1999 « Mass Marketing and Cultural History. The Case of P. T. Barnum », American Quartely, 51, 1, mars $1999: 175-186$.

DELVAU, Alfred

1867 Les Lions du jour, physionomies parisiennes. Paris, Dentu.

DIAS, Nélia

1991 Le Musée d'ethnographie du Trocadéro (1878-1908). Anthropologie et muséologie en France. Paris, éditions du CNRS.

DIPPIE, Brian

1990 Catlin and his Contemporaries. The Politics of Patronage. Lincoln-Londres, University of Nebraska Press.

2001 The Frederic Remington Art Museum Collection. Ogdensburg-New York, Frederic Remington Art Museum.

2004 « Chop ! Chop ! : Progress in the Presentation of Western Visual History ", The Historian, vol. 66,3 , septembre : 491-500.

DONALDSON, Thomas

1886 « The George Catlin Indian Gallery in the U. S. National Museum (Smithsonian Institution) with Memoir and Statistics ", Annual Report of the Smithsonian Institution for 1885, part 5.

Washington, D.C., Government Printing Office.

EATON, Diane, URBANEK, Sheila

1995 Paul Kane's Great Nor-West. Vancouver, University of the British Columbia Press.

EWERS, John C.

1955 « George Catlin : Painter of Indians of the West », Annual Report of the Smithsonian Institution. Washington D.C., U.S. Government Printing Office : 483-528.

FAIRCHILD, Sharon F.

1994 « George Sand and George Catlin. Masking Indian Realities », Nineteenth Century French Studies, vol. XXII, n 364 : 439-449.

FALGUIÈRES, Patricia

2003 Les Chambres des merveilles. Paris, Bayard.

GOODBODY, Bridget Luette

1996 George Catlin's Indian Gallery : Art, Science and Power in the Nineteenth-Century, thèse Ph. D., Columbia University.

GURNEY, George, THAU HEYMAN, Therese, éd. 
2002 George Catlin and His Indian Gallery. Washington, Smithsonian American Art Museum, W. W. Norton \& Company.

JAMIN, Jean

1985 « Les objets ethnographiques sont-ils des choses perdues? », in Jacques Hainard et Roland Kaehr éd., Temps perdu, temps retrouvé. Neuchâtel, Musée d'ethnographie : 51-74.

KANE, Paul

1859 Wanderings of an Artist among the Indians of North America. Londres, Longman.

1861 Les Indiens de la Baie d'Hudson. Promenades d'un artiste parmi les Indiens de l'Amérique du Nord, imité de l'anglais par Édouard Delessert. Paris, Amyot.

KARTUNNEN, Frances

1994 Between Worlds. Interpreters, Guides and Survivors. New Brunswick, NJ Rutgers University Press. KREBS, Edgardo Carlos

1990 « George Catlin and South America : A Look at His "Lost" Years and His Paintings of Northeastern Argentina ", American Art Journal, 22, n $4: 4-39$.

LA HONTAN, Louis-Armand de Lom d'Arce, baron de

2005 (1703), Dialogues curieux entre l'auteur et un Sauvage de bon sens qui a voyagé. Arles, éd. Sulliver.

LAVALLÉE, C.

1859 « Un artiste [P. Kane] chez les Peaux-Rouges », Revue des Deux Mondes, tome 22 : 963-987.

LUSINI, Valentina

2004 Gli oggetti etnografici tra arte e storia. L'immaginario postcoloniale e il progetto del Musée du Quai Branly a Parigi. Turin, L'Harmattan Italie.

MORGAN, Henry Lewis

1959 The Indian Journals, 1859-1862, Leslie White éd. Ann Arbor, The University of Michigan Press.

MOSES, L. G.

1996 Wild West Shows and the Images of American Indians, 1883-1933. Albuquerque, University of New Mexico Press.

MULVEY, Christopher

1987 « Among the Sag-a-noshes : Ojibwa and Iowa Indians with George Catlin in Europe, 1843-1848 », in Christian F. Fest éd., Indians and Europe. An Interdisciplinary Collection of Essays, Aachen, Herodot Verlag : 253-275.

POULOT, Dominique

2005 Une histoire des musées de France. Paris, La Découverte, « L'espace de l'histoire ».

PRICHARD, James Cowles

1843a The Natural History of Man. Londres-New York, Hippolyte Balliere.

$1843 \mathrm{~b}$ Histoire naturelle de l'homme comprenant des recherches sur l'influence des agents physiques et moraux considérés comme cause des variétés qui distinguent entre elles les différentes races humaines.

Paris, J. B. Baillère, 2 vol.

REDDIN, Paul 
1999 Wild West Shows. Urbana, University of Illinois Press.

ROTSCHI, Agnès

1993 «Les œuvres de George Catlin conservées au Musée de l'Homme », Musées/Homme, n 1 :

38-39.

STOCKING, George W.

1973 « From Chronology to Ethnology : Prichard and British Anthropology, 1800-1850 », in James Cowles Prichard, Researches into the Physical History of Man. Chicago, The University of Chicago Press, « Classics in Anthropology ».

SWEENEY, J. Gray

2003 « Inventing Luminism : “The Labels are the Dickens" », Oxford Art Journal, 26, 2 : 93-120.

TRUETTNER, William H.

1979 The Natural Man Observed : A Study of Catlin's Indian Gallery. Washington D.C., Smithsonian Institution Press.

TRUETTNER, William H., ANDERSON, Nancy K., éd

1991 The West as America : Reinterpreting Images of the Frontier, 1820-1920. Washington D. C., Smithsonian Institution Press.

WILLIAMS, Élisabeth A.

1985a « Arts and Artifacts at the Trocadero. Ars Americana and the Primitivist Revolution », in Stocking George W. Jr, éd., Objects and Others. Essays on Museums and Material Culture. Madison, University of Wisconsin Press : 146-166.

$1985 b$ « Anthropological Institutions in Nineteenth Century France », Isis, vol. 76 : 331-348.

WILSON, Daniel

1892 The Lost Atlantis and Other Ethnographic Studies. Édimbourg, Douglas.

\section{NOTES}

1. Outre le catalogue de l'exposition récente (Gurney et Thau Heyman 2002) qui contient la bibliographie essentielle (p. 282-285), il faut se reporter à Brian W. Dippie (1990). Sur les voyages sud-américains des années 1850, Carlos Edgardo Krebs (1990) a réalisé une belle enquête.

2. Voir Donaldson 1886. L'ensemble de la correspondance suscitée par ce travail est conservé à la Smithsonian Institution.

3. Telle est la signature de Catlin comme auteur et comme personne, voir plus loin le commentaire proposé par Claude Macherel.

4. Voir Truettner 1979 et Truettner et Anderson 1991.

5. Sur son œuvre, voir Dippie 2001.

6. En ce sens, Catlin pourrait prendre place dans la cohorte des médiateurs identifiés par Frances Karttunen (1994).

7. C'est la thèse de Reddin 1999, dont la rédaction est très antérieure. Sur la période où fut véritablement inventé le West Wild Show, à partir des années 1880, voir Moses 1996.

8. Dominique Poulot (2005) a tracé la généalogie du musée moderne. Patricia Falguières (2003) met en évidence l'héritage des chambres des merveilles.

9. Voir plus loin l'article de Daniel Fabre. 
10. Sur la genèse et les formes du spectacle de masse aux États-Unis, voir Bogdan 1988, Adams 1997 et le commentaire de Cook 1999. Lire aussi les mémoires (1873) et la correspondance (1983) de Barnum qui précise à diverses reprises ses rapports avec Catlin.

11. Six Osages, venus de Louisiane dans l'intention de protester contre la vente par la France de cette région en 1803, sont reçus à Paris par Charles $\mathrm{X}$, déclenchent un engouement extraordinaire (voir Delvau 1867 : 37-39), suscitent des images (voir plus loin l'article de Frédéric Maguet) avant d'être abandonnés à une misérable errance, prélude à leur réembarquement. Sur cette histoire et ses lendemains, voir le site de l'association montalbanaise OK/OC. Nous remercions Michel Melot de nous avoir mis sur cette piste.

12. Voir l'ouvrage collectif Bancel... 2002.

13. Dippie 1990 : 102-105.

14. La première étude innovante sur les troupes indiennes de Catlin est de Christopher Mulvey 1987 ; des compléments sont apportés par Fairchild 1994 et Buddle 2004. Nous avons depuis découvert d'autres sources qui pourraient fonder une analyse beaucoup plus complète des conditions de leur départ et de leur retour.

15. Voir plus loin l'article de Claude Macherel sur le «syndrome de Noé » qui oriente toute l'existence de Catlin.

16. La Hontan 2005 (1703); Montesquieu 1721.

17. C'est en 1848 que Catlin modifie ainsi le titre du Catalogue qu'il ne cessait de remettre à jour.

18. Sur ces rivalités, l'apport de Dippie (1990) est essentiel.

19. Cette contribution n'a pas attiré l'attention des spécialistes. Prichard est pourtant un des fondateurs de l'anthropologie conçue comme histoire naturelle de l'homme incluant les faits de culture. Voir la longue présentation de George W. Stocking (1973) à la réédition de son premier livre.

20. Voir Wilson 1892 (cit. Catlin : 123-124).

21. Sur ce champ de l'ethnologie naissante à Paris, voir Williams 1985a et b; Dias 1991 ; BrescBautier 1999 et Lusini 2004.

22. Chacune de ces relations savantes européennes a laissé ses traces imprimées et manuscrites, encore vierges de toute recherche.

23. L'O-kee-pa a été publié sitôt écrit, en 1865, dans une édition londonienne pirate et deux ans plus tard par Catlin lui-même. Les deux manuscrits (sur l'o-kee-pa et Pipe stone) réalisés par Catlin et par lui vendus au British Museum n'ont été découverts et publiés qu'assez récemment (Catlin 1979 et 1996).

24. Morgan 1959 : 71-81 et 157-158.

25. Cette réception de la peinture fait l'objet de l'article plus loin de Daniel Fabre.

26. Voir sur ces reclassements esthétiques et nationaux : Sweeney 2003 et Dippie 2004.

27. Le commentaire du Bateau ivre comme transposition d'articles du Magasin pittoresque ou de L'Illustration a été tenté plusieurs fois. Ajoutons à ce dossier la parution en mai 1869, dans Le Tour du monde de deux articles («Excursions parmi les tribus indiennes des bassins de la Columbia... » et «L'O-kie-pa... » (sic)) où l'on pourrait aussi bien reconnaître des images du périple rêvé de Rimbaud.

28. Dippie 1990 : 142-143.

29. Voir Le Canada 1855 ; Kane 1859 et 1861 ; Lavallée 1859 ; Eaton et Urbanek 1995.

30. Le fait est parfaitement exact, mais il élimine tout le reste.

31. L'expression est de Georges Henri Rivière et elle énonce le rêve qui l'a longtemps porté. 
AUTEUR

DANIEL FABRE 